\title{
The lineshape of inelastic neutron scattering in the relaxor ferroelectrics
}

\author{
(C) M.A. Ivanov, M. Kozłovski*, T. Piesiewicz*, V.A. Stephanovich**, A. Weron ${ }^{* * *}$, A. Wymysłowski**** \\ Institute of Metal Physics, Ukranian National Academy of Sciences, Kiev, Ukraine \\ * Institute of Power Systems Automation \& Laboratory for Interconnecting and Packaging Electronic Circuits, \\ Wrocław University of Technology, Wrocław, Poland \\ ** Opole University, Institute of Mathematics and Informatics, Opole, Poland \\ *** Hugo Steinhaus Center for Stochastic Methods, \\ Wrocław University of Technology, Wrocław, Poland \\ **** Laboratory for Interconnecting and Packaging Electronic Circuits, \\ Wrocław University of Technology, Wrocław, Poland \\ E-mail: ivanov@imp.kiev.ua \\ Tomasz.Piesiewicz@pwr.wroc.pl \\ stef@math.uni.opole.pl \\ weron@im.pwr.wroc.pl \\ Artur.Wymyslowski@pwr.wroc.pl
}

(Received 4 November 2004

Revised 6 Februari 2005)

\begin{abstract}
We show that a microscopic reason for a steep dropping of the optical phonon branch into the acoustic one (the so-called waterfall effect) in relaxor ferroelectrics may be the coupling of phonons with the defects and impurities of different kinds, which is always present in relaxors. Namely, we do not specify the type of impurities but rather represent them as an ensemble of so-called two-level systems (TLS). This approach permits to trace the evolution of the „waterfall" with temperature and the TLS concentration. To facilitate the planning of experiments on inelastic neutron scattering, we present a modification of the so-called Latin Hypercube Sampling method, which, based on some significance criteria, permits, to perform the measurements which are the most signeficant to elucidate the physical nature of, e.g., phonon dispersion laws in relaxor ferroelectrics.
\end{abstract}

Partial financial support was given to this work by the European Community 5th Framework Programme (MEVIPRO Project).

\section{Introduction}

The advantages of the neutron scattering method (as compared to, e.g., Raman spectroscopy) in the investigation of ferroelectrics and antiferroelectrics are well known [1,2]. Since the wavelenght of neutrons equals (by order of magnitude) to the lattice constant of a solid and their energy and momentum is comparable to that of phonons, the neutron scattering permits to obtain the information inaccessible by other (e.g. optical) methods. For instance, it is possible to obtain the dependence of phonon energy on its momentum known as its despersion law. Inelastic neutron scattering permits to obtain the detailed information about soft phonon mode (and actually any other phonon mode) dispersion throughout entire Brillouin zone. Such investigations for relaxor ferroelectric $\mathrm{PbMg}_{1 / 3} \mathrm{Nb}_{2 / 3} \mathrm{O}_{3}$ have been performed, e.g., in [3]. It is also possible to study the thermal displacements of ions from their equilibrium positions in the elementary cell, also during ferroelectric phase transition. Besides that the inelastic neutron scattering is free from limitations imposed by the optical selection rules.

Recently, much attention has been paid to the investigation of phonon dispersion in relaxor perovskites. The main peculiar (and puzzling) feature here is the so-called „waterfall effect" or very steep dropping of the optic phonon branch into acoustic one at some value $q_{\mathrm{wf}}$ of momentum transfer. This effect has been first observed in [4] and $q_{\mathrm{wf}}$ was interpreted as a characteristic wave vector corresponding to the reciprocal size of so-called polar nanoclusters (in other words, short range ordered polar clusters), which prevent the optical phonons with $q<q_{\mathrm{wt}}$ to propagate in a lattice.

In the disordered ferroelectrics, the size of the above nanoclusters is defined by the so-called correlation radius. Latter quantity, being the characteristic length of the fluctuations of polarization, is a characteristic feature of any ferroelectric (antiferroelectric) substance, see, e.g. [5]. The correlation radius grows infinitely near the ferroelectric (antiferroelectric) phase transition temperature. The knowledge of the soft mode dispersion law at different temperatures permits to extact the temperature dependence of the correlation radius. Measurements of the correlation radius in the $\mathrm{PbMg}_{1 / 3} \mathrm{Nb}_{2 / 3} \mathrm{O}_{3}(\mathrm{PMN})$ relaxor $[6,7]$ revealed its peculiar temperature dependence: a plateau at $T \leq T_{g}$ ( $T_{g}$ is the freezing temperature of the dipole glass state) and another plateau at $T \geq 400 \mathrm{~K}$.

The waterfall effect was later observed in a number of relaxors [8] and described in terms of coupled optical and acoustic phonon branches in [9]. The authors of [9] based their explanation on the model, suggested in the earlier work [10]. Their key assumption was the drastic increase of the damping of the „bare“ (i.e. before coupling) optical phonon branch at $q \leq q_{\mathrm{wf}}$. Another explanation of the waterfall effect was proposed in [11], which uses essentially the same approach as above (i.e. the mode coupling model), but does not attribute $q_{\mathrm{wf}}$ to 
the size of polar nanoclusters. In other words, the authors of [11] do not assume the sudden change of optical mode damping at $q \leq q_{\mathrm{wf}}$, but rather use a model of two coupled damped harmonic oscillators representing optic and acoustic branches. The coefficients of the corresponding „bare“ dispersion laws as well as of damping have been taken phenomenologically from the best fit to experiments.

All above approaches do not take into account the influence of the ensemble of defects and impurities (inherent to any disordered ferroelectric and relaxor in particular) on the phonon dispersion law. One of the aims of the present paper is to suggest a formalism, permitting to do that. The main physical idea is that impurities and defects of different kind (for instance, impurity dipoles) interact with each other indirectly via one of the transversal optical phonon branches of a host dielectric. Due to this interaction, the above mode by itself softens (i.e. its frequency being renormalized by the impurities becomes zero at some temperature and generally speaking wave vector $q$ ) so that the ferroelectric phase transition may occur. This mechanism had been shown to be the origin of ferroelectricity in the incipient ferroelectrics [12]. The long wavelength dispersion of the soft phonon mode of such system was essentially renormalized by the impurities, as compared to the case of host incipient ferroelectric. This renormalized dispersion law defines the width of the distribution function of random fields (created by the impurity ensemble [13]) that influences all the observable physical quantities of the disordered ferroelectrics. The static and dynamic properties of the disordered systems have been attributed to and expressed via the correlation radius of a host lattice (see, e.g. $[5,14,15]$ ). The host lattice is $\mathrm{KTaO}_{3}$ in the case of $\mathrm{K}_{1-x} \mathrm{Li}_{x} \mathrm{TaO}_{3}$ (KTL) [12] and Burns reference phase in the case of relaxor ferroelectrics [16].

The experimental investigations of relaxor ferroelectrics by inelastic neutron scattering method are dependent on many parameters (see, e.g. $[3,6,7]$ ) like temperature, concentrations of different kinds of defects and impurities (sometimes called degree of disorder), vectors of the reciprocal lattice etc. To draw the correct conclusions from such multifactor experiment, we should perfectly know, which measurements (i.e. for which parameter values) give the most significant information for the system considered. Additional factor here is that the neutron scattering experiments are quite costly and corresponding equipment is present in very small number of laboratories. So, the best way to improve the effectiveness of neutron scattering experiments is to plan them in advance. Usually, the task of multifactor experiment planning is performed by the so-called Latin Hypercube Sampling (LHS) method, first introduced by McKay et al. [17]. In this method, the space of $(n)$ experimental parameters is represented as $n$-dimensional Euclidean space, where the point correspond to each specific measurement performed with certain set of above parameters. In the standard LHS method, all the parameters are supposed to be of the same significance so that the number of possible experiments is too high to be performed in reasonable time. Here we present a modification of the LHS method, which, based on some significance criteria, permits to perform the measurements which are the most significant to elucidate the physical nature of, e.g., phonon dispersion laws in relaxor ferroelectrics.

\section{Neutron scattering lineshape of the disordered dielectric}

It is well-known (see, e.g. $[2,18]$ ) that the differential cross section of the neutron scattering is proportional to a Fourier image of the density-density correlation function

$$
S(\mathbf{K}, \omega)=\int \mathscr{P}(\mathbf{r}, t) \exp [i(\mathbf{K r}-\omega t)] d^{3} r d t,
$$

where

$$
\mathscr{P}(\mathbf{r}, t)=\int\left\langle\rho\left(\mathbf{r}^{\prime}, 0\right) \rho\left(\mathbf{r}^{\prime}+\mathbf{r}, t\right)\right\rangle d^{3} r .
$$

Here angular brackets mean the quantum-statistical averaging, $\hbar \mathbf{K}$ is a vector of a scattered neutron momentum, $\hbar \omega=\hbar^{2} K^{2} / 2 m_{k}$ is its energy.

The scattering density $\rho(\mathbf{r}, t)$ can be expressed in the form

$$
\rho(\mathbf{r}, t)=\sum_{i} b_{i} \delta\left(\mathbf{r}-\mathbf{r}_{i}(t)\right),
$$

where $b_{i}$ is a scattering length for the $i$-th nucleus localized at the point

$$
\mathbf{r}_{i}(t)=\mathbf{r}_{l k}+\mathbf{u}_{l k}(t),
$$

where $\mathbf{r}_{l k}$ is the equilibrium position of the $k$-th atom in the $l$-th elementary cell, $\mathbf{u}_{l k}(t)$ is its displacement. If this displacement is due to lattice vibrations, we can expand it over the phonon normal coordinates in the following way

$$
\mathbf{u}_{l k}(t)=\left(\frac{\hbar}{2 N m_{k}}\right)^{1 / 2} \sum_{\mathbf{q}, j} \lambda_{k j}(\mathbf{q})\left[Q_{j}(\mathbf{q}, t) \exp \left(i \mathbf{q} r_{l k}\right)+c . c .\right] \text {. }
$$

Here $Q_{j}(\mathbf{q}, t)$ is the normal coordinate of the phonon for the $j$-th branch of the phonon spectrum, $\lambda_{k j}$ is the polarization of the phonon, $\mathbf{q}$ is its wave vector related (by virtue of momentum concervation) to the wave vector $\mathbf{K}$ of scattered neutron

$$
\mathbf{K}+\mathbf{q}=\boldsymbol{\tau},
$$

where $\boldsymbol{\tau}$ is a reciprocal lattice vector. Note that most often the neutron scattering measurements in relaxors are performed for $\boldsymbol{\tau}=(2,0,0)$ and $(3,0,0)$ (see, e.g. $[3,8]$ ). We will use this fact below for planning experiments by the LHS method.

The expression for $S(\mathbf{K}, \omega)$ assumes the following form with respect to relations $(3)-(5)$

$$
S(\mathbf{K}, \omega)=N \sum_{\mathbf{q} j}\left|F_{j}(\mathbf{K})\right|^{2} \int \Phi(\mathbf{q}, t) \exp (i \omega t) d t,
$$

where $F_{j}(\mathbf{K})$ is a structural factor of inelastic scattering and $\Phi(\mathbf{q}, t)=\left\langle Q_{j}(-\mathbf{q}, 0) Q_{j}(\mathbf{q}, t)\right\rangle$ is the phonon (of the $j$-th branch) displacement correlation function. 
Since the experimentally sensitive quantity is just above correlator, here we are not interested in the precise form of $F_{j}(\mathbf{K})$. There are many ways to calculate the correlator. Here, in order to capture the waterfall effect, we will use the model of two coupled oscillators, being optical and acoustic modes. In this paper, trying to keep everything as simple as possible, we assume that only optical mode is coupled to the impurity ensemble and this coupling is the source of its damping.

The fluctuation-dissipation theorem relates the function $S(\mathbf{K}, \omega) \equiv S(\mathbf{q}, \omega)$ to the imaginary part of the retarded phonon Green's function $\hat{G}(q, \omega)$, which in the case of two coupled oscillators will be a $2 \times 2$ matrix. More precisely

$$
S(q, \omega)=\frac{T}{\omega} \mathbf{F}(q)^{*} \operatorname{Im}[\hat{G}(q, \omega)] \mathbf{F}(q),
$$

where $\mathbf{F}(q)$ is a structure factor,

$$
\hat{G}^{-1}(1, \omega)=\left(\begin{array}{cc}
\omega^{2}-\omega_{T A}^{2} & \Delta \\
\Delta & \omega^{2}-\omega_{T O}^{2}-\Pi_{1}-i \Pi_{2}
\end{array}\right),
$$

$\Pi^{*}=\Pi_{1}+i \Pi_{2}$ is a self-energy, which we write in the form customary for interaction of phonons with two-level systems (TLS) [19], namely

$$
\Pi^{*}=\alpha_{0} \int_{0}^{\omega_{m}^{2}} \frac{P(\lambda) d \lambda}{\omega^{2}+i \Gamma \omega-\lambda} .
$$

Here $\alpha_{0}$ is a phonon-TLS coupling coefficient, $\Gamma$ defines the damping of optical phonons due to their interaction with TLS $[23]^{1}$ and $P(\lambda)$ is a TLS density of states. The value $\omega_{m}^{2}=c \omega_{D}^{2}\left(\omega_{D}\right.$ is Debye frequency $)$ defines the width of the distribution function $P(\lambda)$ so that it is normalized by the condition

$$
\int_{0}^{\omega_{m}^{2}} P(\lambda) d \lambda=c
$$

where $c=N_{\mathrm{TLS}} / N$ is a dimensionless concentration of TLS, defined as a ratio of the TLS number $N_{\mathrm{TLS}}$ to the host lattice particle number $N$, so that $0<c<1$. We take $P(\lambda)$ in a simple form

$$
P(\lambda)=\frac{c}{\ln 2} \frac{1}{\omega_{m}^{2}+\lambda} .
$$

To calculate the intensity $S(q, \omega)$, we should now evaluate integrals (9) with respect to (11) and substitute the dispersion laws for optical and acoustic phonons into inverse matrix (8). We take the dispersion laws in long-wavelength limit

$$
\begin{gathered}
\omega_{T A}^{2}=s_{0}^{2} k^{2}, \\
\omega_{T O}^{2}=a\left(T-T_{c}\right)+b k^{2},
\end{gathered}
$$

where we take $T_{c} \approx 400 \mathrm{~K}$ from [3]. Now integrals (9) can be easily evaluated. Their explicit form is quite cumbersome so we do not cite it here.

\footnotetext{
${ }^{1}$ We do not consider here the imaginary part of $\Pi^{*}$ (9) (namely $\left.\Pi^{*}=\alpha \int_{0}^{\omega_{m}^{2}} \frac{P(\lambda) d \lambda}{\omega^{2}+i 0-\lambda}\right)$ due to the Lifshitz tails of the density of states as it is much smaller then its intrinsic damping term.
}

The specific calculations have been performed for the following set of dimensionless variables

$$
\begin{gathered}
y=\frac{\omega}{\omega_{D}}, \quad y_{T A, T O}=\frac{\omega_{T A, T O}}{\omega_{D}}, \quad \tau=\frac{T}{T_{c}}, \\
\kappa=\frac{a T_{c}}{\omega_{D}^{2}}, \quad q=\sqrt{\frac{b}{2}} \frac{k}{10 \omega_{D}}, \quad s=\frac{10 s_{0} \sqrt{2}}{\sqrt{b}}, \\
\alpha=\frac{\alpha_{0}}{\omega_{D}^{4}}, \quad \gamma=\frac{\Gamma}{\omega_{D}}, \quad \delta=\frac{\Delta}{\omega_{D}^{2}} .
\end{gathered}
$$

Keeping in mind that in (12) $a\left(T-T_{c}\right) \propto 1 / \chi, \chi$ is a dielectric susceptibility of relaxor, it can be shown that $\kappa \sim 1$. Also, $s \sim 1$. We perform our specific calculations for a set of following values: $\alpha=0.8, \kappa=1, s=2, \delta=10 q^{2}$, $\gamma=2$. The shape of intensity profiles as functions of $k$ and $\omega$ are qualitatively similar to those from [8]. The most interesting features reveal themselves in the dispersion curve $\omega(k)(y(q)$ in dimensionless variables), obtained by usual procedure of taking the positions of maximum intensity (7) for several constant $\omega$ values.
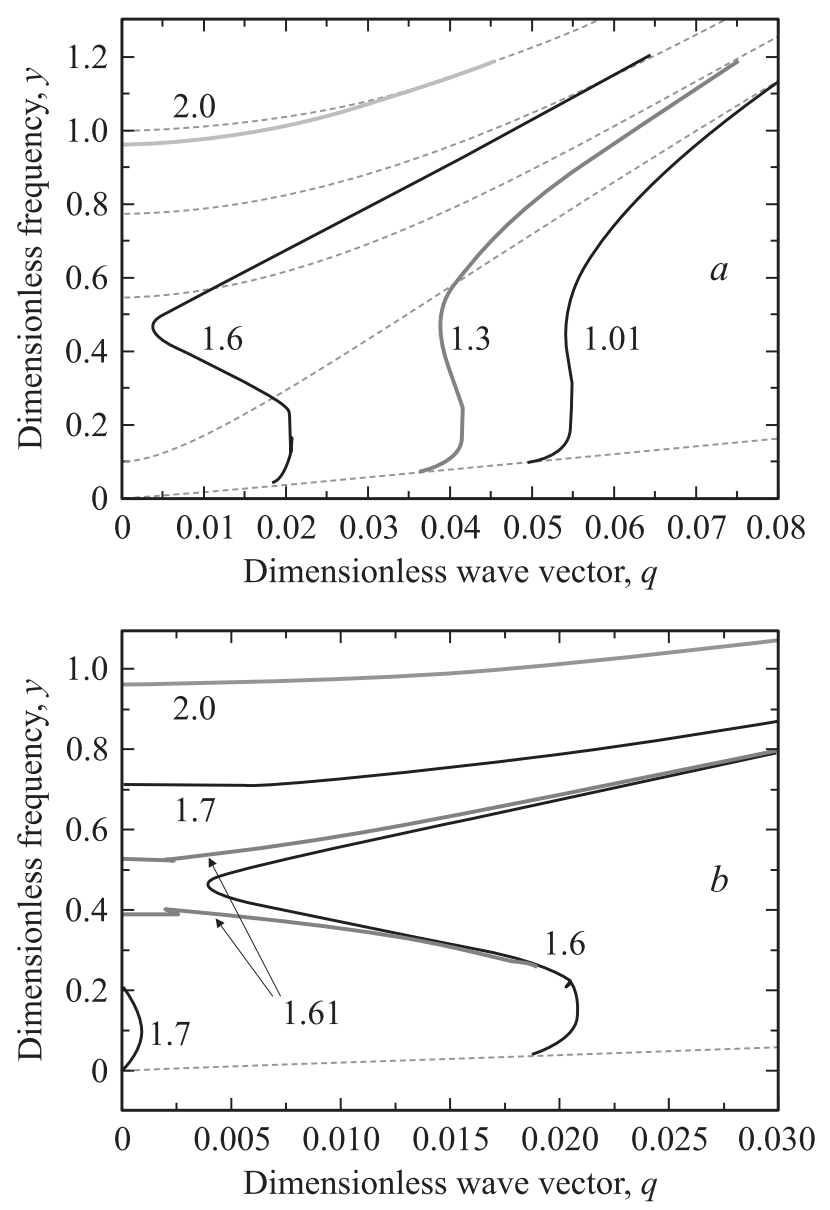

Figure 1. Dispersion curves calculated for different temperatures (thick lines) for $\mathbf{F}=(0.2,0.8)$ and $c>0.4$. $a-$ general view for larger temperature step, $b$ - detailed wiew, showing the "decay“ of the „waterfall“. Figures near curves correspond to values of the dimensionless temperature $\tau$. Thin dashed lines correspond to the frequencies of coupled optical and acoustic modes for corresponding temperatures. 


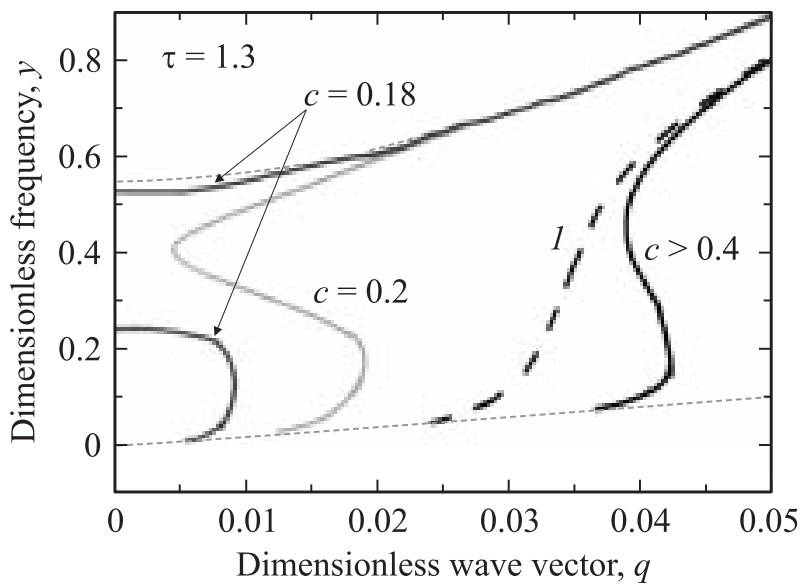

Figure 2. Dispersion curves calculated for different $c$. Here $\tau=1.3, \mathbf{F}=(0.2,0.8)$. Thin dashed lines are coupled optical and acoustic modes frequencies. Curve 1 corresponds to the same values (i.e. $c>0.4)$ and $\mathbf{F}=(0.5,0.5)$.

The above curves $y(q)$ are reported in Fig. 1 for different temperatures. It is seen from Fig. 1 that at temperatures close to $T_{c}$ the $y(q)$ curves almost vertically (with very small s-shape) rise towards (coupled with acoustic) corresponding optical branch. At higher temperatures this ,jump“ is more s-shaped and at some temperature (around 1.61 in our case, see Fig. 1,b) „splits“ into two parts, one of them beins close to the acoustic and other to optical modes. At further increasing of temperature the „acoustic" part degenerates into a small segment, which disappears at $\tau \approx 1.71$. For higher temperatures instead of the waterfall we have separete (although coupled by virtue of $\delta$ ) acoustic and optical branches. It should be noted here, that the parts of s-shaped curves $y(q)$, where $d y / d q<0$, correspond to the minimun of corresponding constant energy curves. This means that two „waterfalls“ (i.e. a sharp drop or increase in the corresponding dispersion curve) can be observed experimentally by increasing or decreasing the wave vector $q$. Possible experimental observation of such „waterfall hysteresis“ may be an argument in favor of the present model and contrary, the absence of the effect would demonstrate that feature is just an artifact of it. Note also that here we adjust the rest parameters in such a way, that waterfall disappears around $T_{0} \approx 1.6 T_{c}=640 \mathrm{~K}$, which corresponds to Burns temperature for $\mathrm{PbMg}_{1 / 3} \mathrm{Nb}_{2 / 3} \mathrm{O}_{3}$.

The above dispersion curves are also concentrationally dependent. This dependence is shown in Fig. 2. It is seen that, while lowering the concentration, the waterfall behaves qualitatively similar to that while rising the temperature. Also, at very small concentrations there are two distinct phonon branches without any waterfall effect. This is due to the simple fact that, at $c \rightarrow 0, \Pi^{*} \equiv 0$ so that there is no imaginary part in the matrix $\hat{G}$.

Curve 1 in Fig. 2 shows the curve $y(q)$ for different bare structural factor $F=(0.5,0.5)$. It is seen that the position of the waterfall $q_{\mathrm{wf}}$ remains almost unchanged, while the s-shape disappears. Our analysis shows that the $\mathrm{s}$-shape is regained at higher temperatures or at smaller concentrations, while $q_{\mathrm{wf}}$ will follow the corresponding value for $\mathbf{F}=(0.2,0.8)$.

\section{The optimization of planning of experiment by modified LHS method}

To draw more specific conclusions about vibrational spectra of relaxors, it is necessary to perform many experiments to extract reliable phonon dispersion curves from above inelastic neutron scattering lineshapes. Here we are going to present the modified LHS method to plan such experiments.

In the LHS method (see e.g. [17,20-24]), the range of all $d$ input variables (i. e. temperature, impurity concentrations etc in our case) is divided into $n$ intervals, $n$ being the number of sampling points. Each interval for each variable is randomly chosen only once with equal probability. A sampling point is chosen within each interval using the uniform probability density. Such procedure prevents the formation of the sampling points clusters.

Formally, suppose that $x_{i, j}(i=1 \ldots n, j=1 \ldots d)$ is the $i$-th value of the $j$-th variable (i.e. experimental parameter), $n$ being the number of sampling points and $d$ being the number of input variables. Let us define $p_{i, j}$ as the $n+d$ matrix, each column being an independent random permutation of the set $(1 \ldots n)$. Moreover, let $u_{i, j}$ be $n+d$ values of the uniform $[0,1]$ independent random variables. The LHS poinst are defined as

$$
x_{i, j}=\frac{1}{n}\left(p_{i, j}-u_{i, j}\right) .
$$

In this case, the points are well scattered and do not form clusters because observations are restricted within the respective interval. Moreover, it is possible to run the $\mathrm{LH}$ sampling procedure many times to maximize the minimal distance between two points in the generated sample.

Our modification of the LHS method is to apply the probability density functions (PDF) to change the distribution of the parameters of experiment. Suppose that $x_{i, j}$ is a standard LH sample and $F_{j}(x)$ is a probability function (for the $j$-th variable) corresponding to the probability density function (or distribution function) $f_{j}(x)$. We define the modified LH sample as

$$
x_{i, j}^{\bmod }=F_{j}^{-1}\left(x_{i, j}\right) .
$$

Our extensive numerical modelling of the LHS planning of experiments in different physical systems show that very accurate adjustment of experimental parameters can be accomplished by following three parameters PDF

$$
f(x)= \begin{cases}b+(1-b)(p+1)\left(\frac{x}{a}\right)^{p}, & x \in(0, a] \\ b+(1-b)(p+1)\left(\frac{1-x}{1-a}\right)^{p}, & x \in(a, 0),\end{cases}
$$

where: $a \in(0,1)$ affects the position of the position of the peak of function $f(x), b \in[0,1+1 / p]$ affects the elevation 

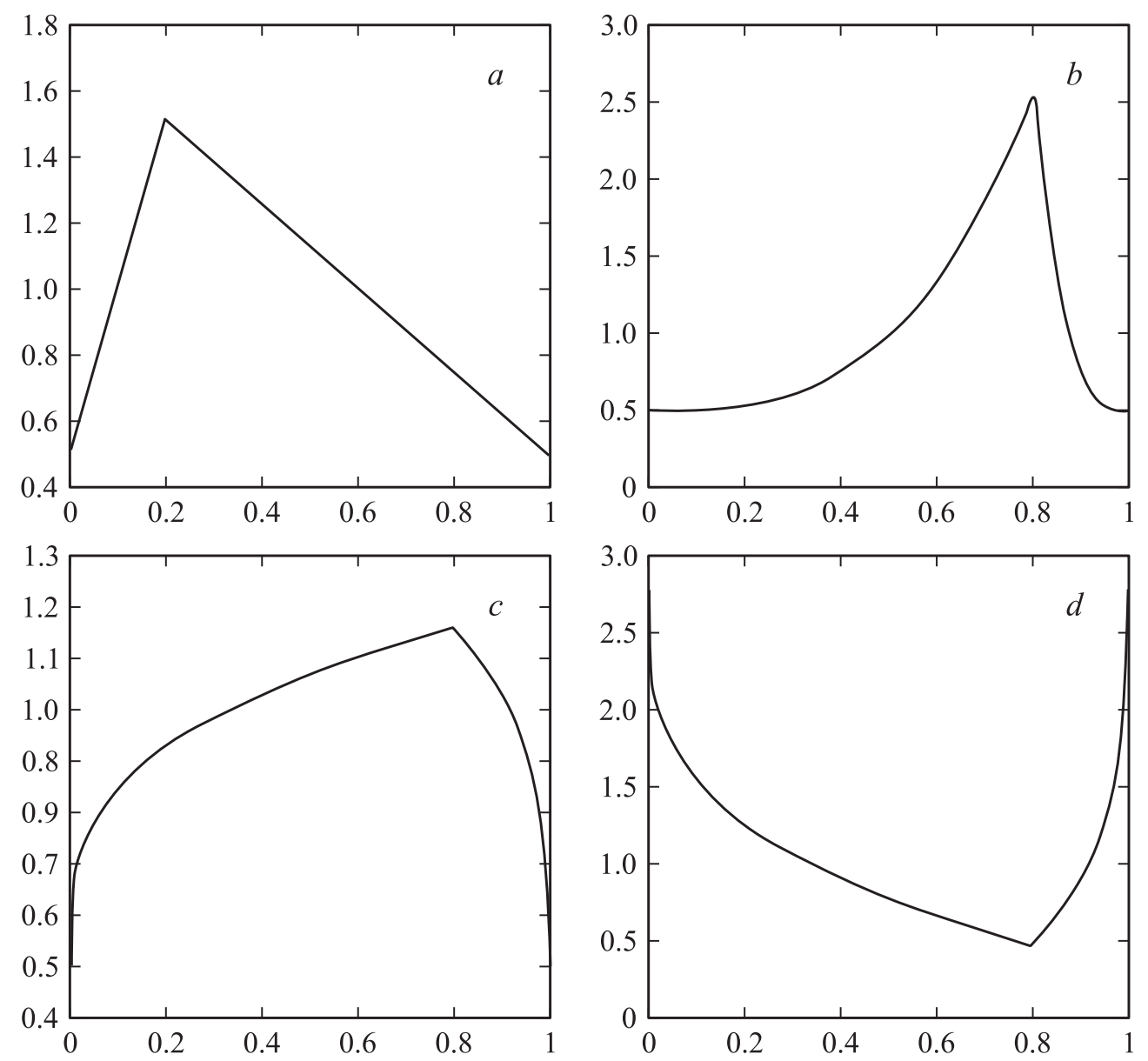

Figure 3. Examples of the density function for various parameters $a, b$ and $p$ : $a-a=0.2, b=0.5, p=1 ; b-a=0.8, b=0.5$, $p=3 ; c-a=0.8, b=0.5, p=0.3 ; d-a=0.8, b=2.8, p=0.3$.

of the peak and $p \in[0, \infty)$ affects the strength of the curve. For $a=0$ or $a=1$ the $f(x)$ in (16) is undefined, so we have to use boundary PDF instead

$$
\begin{aligned}
& f(x)=b+(1-b)(p+1)(1-x)^{p}, \quad a=0, \\
& f(x)=b+(1-b)(p+1) x^{p}, \quad a=1 .
\end{aligned}
$$

For $b=0.5, p=1$ (and any $a$ ) we have standard triangle distribution. Increasing the parameter $p$ causes stronger concentration of the PDF points in the area appointed by parameter $a$. Decreasing the parameter $p$ weakens the concentration. If $b>1$, we obtain the dispersion of PDF points rather then their concentration. For $b=1$ or $p=0$, Eq. (16) gives the standard uniform PDF in $[0,1]$, $f(x)=1$. To visualize the above behavior of $f(x)$, we plot its shape for different values of parameters in Fig. 3. Thus, adjusting the $a, b$ and $p$ parameters allows us to modify the distribution of the points freely, and therefore to include the knowledge of an expert into the LH sampling method. Such knowledge in our case is, for example, the choice of the proper reciprocal lattice vector $\boldsymbol{\tau}$ (in [3], it is $(3,0,0)$ ) to clearly observe the soft mode. In particular, the authors of [3] have shown that observation of the soft phonon mode in PMN is much easier (as compared to previous results performed in the Brillouin zone centered at $(2,0,0)$ ) in the [010] direction near the $(3,0,0)$ reciprocal lattice vector.

The next modification we propose is to allow a researcher to indicate areas he wants to put a sampling point in. On the other hand, it is possible to exclude certain areas from the sampling procedure. Including (excluding) areas is only allowed if there is still possibility to generate a proper LH sample, e.g. it is forbidden to indicat more than one area in the same row or to exclude all column. The information about all indicated areas is stored in the structure we call the ,expert matrix“. After generating the LH sample (see Fig. 4) the experiments should be done to obtain the results. Then the surface of response parameters (response surface) is created by interpolating the results throughout the desured area. The surface is then used for further analysis, e.g. optimization procedures.

Our comparison of the standard and modified LHS methods according to several criteria show that the modified LHS method substantially decreases the number of measurements, necessary to achieve our goal. Latter method is especially useful when it is possible to include the expert knowledge (see above) into consideration, see Appendix for details. 

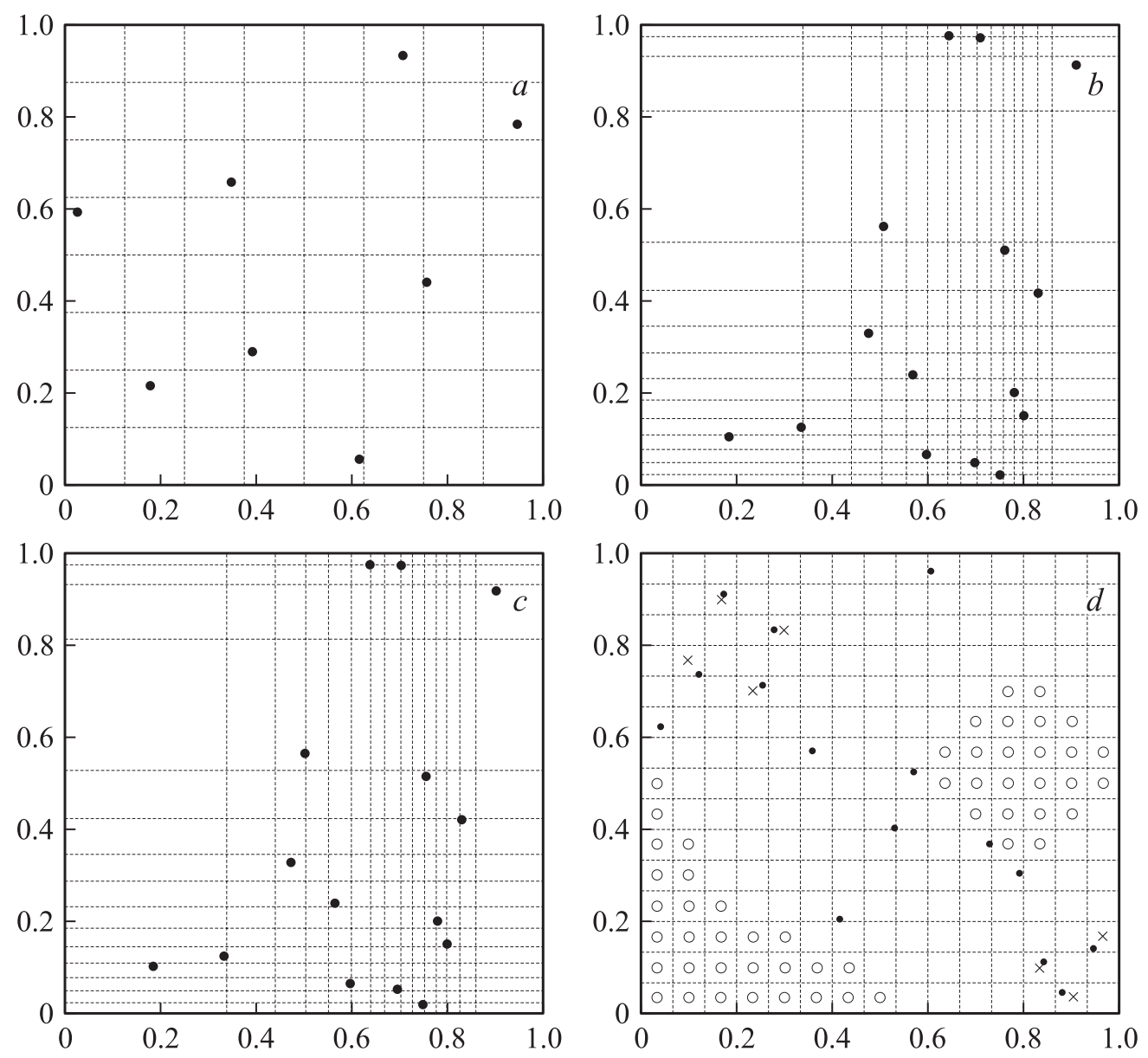

Figure 4. Standard $(a)$ and modified $(b-d)$ LHS grids. In part $a n=8, d=2$, parts $b, c$ correspond to $n=15, d=2$ and parameters $a, b$ and $p$ from Fig. 3, $b$ and $d$ respectively. Fig. 4, $d$ corresponds to the modified LHS grid with the „expert matrix“, the areas marked with crosses include sampling points and those marked with open circles are omitted in the sampling procedure.

\section{Conclusion}

In the present paper we have considered the possibilities of theoretical and experimental investigations of relaxor ferroelectrics by inelastic neutron scattering method. We suggest a simple model which permits to describe the peculiarities of inelastic neutron scattering lineshapes in ferroelectric relaxors. The essence of our model is to consider the interaction of the phonon subsystem of relaxor ferroelectrics with the ensemble of defects and impurities which are always present in such cubstance. This interaction has been taken into account in quite general yet simple form without consideration of detailed nature of defects and impurities. Our simple approach permits to obtain temperature and concentrational dependences of the so-called waterfall effect, which recently has attracted much attention of scientists. Our dependencies of corresponding dispersion laws on temperature and the impurities concentration are in a qualitative agreement with available experimental data (see, e.g. $[8,9,11]$ ). Unfortunately, to make more specific comparison, we need to take into account many additional factors, for instance the acoustic phonon damping. Even in our simple model, the problem remains highly multiparametric - we have six parameters of the model additionally to the temperature and concentration. Small variation of several above parameters may cause very large diffenences in behaviour of calculated $S(q, \omega)$. To have better parameters of our theory (such as $\kappa, s_{0}$ etc), more experiments on relaxors aiming to obtain, e.g., the precise values of coefficients in phonon dipersion laws, should be performed. Also, other approaches can be used as complementary ones to calculate $S(q, \omega)$. One of them is the so-called random field method, which has already been applied for calculation of the correlation radius in relaxors [5]. The latter calculation is also suitable to calculate the renormalized (by dipole impurities) dispersion law of the soft phonon mode in relaxors. The inclusion of other types of impurities (like elastic dipoles and point electric charges) into consideration will permit to estimate their partial contribution into the peculiarities of neutron scattering in relaxor, e.g. in waterfall effect.

Also, we suggest that to optimize the (multifactor) experimental investigations of relaxor ferroelectrics by inelastic neutron scattering method one may use the modified LHS method. The essence of modification is the introduction 
of empirical PDF (16) which permits to include the „knowledge of an expert" into consideration.

We note finally, that to distinguish between different microscopic physical mechanisms, leading to appearance of peculiarities of neutron scattering in relaxors like the discussed waterfall effect, the experiments, elucidating the specific features of a certain theoretical model (like presence or absence of a s-shape on the waterfall curve), are highly desirable.

\section{Appendix A}

In this paragraph we will compare the standard and modified LHS method according to several criteria. At first we will generate standard LH sample and modified LH sample the size of $10,15,20,25,30,35$ and 40 points. To properly count the errors of response surface adjustment we will take as a results an analytical function with single extreme $\left(f_{1}\right.$, see Fig. 5, $\left.a\right)$ and, in second case, a function with two separate maxima located on the boundaries - one of them a bit higher than the other $\left(f_{2}\right.$, see Fig. 5, $\left.b\right)$.
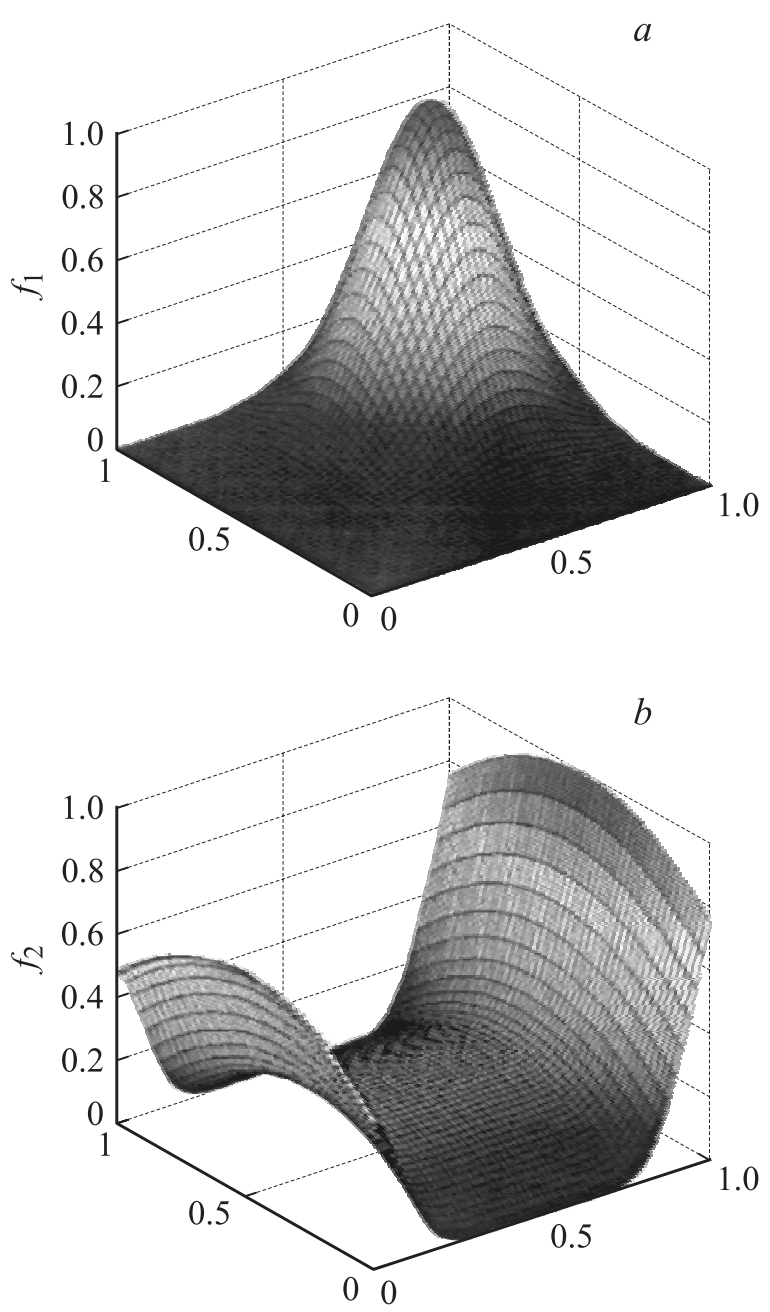

Figure 5. Functions used in tests $(a)$ function $f_{1}$ with a single maximum; $(b)$ function $f_{2}$ with two separate maxima.
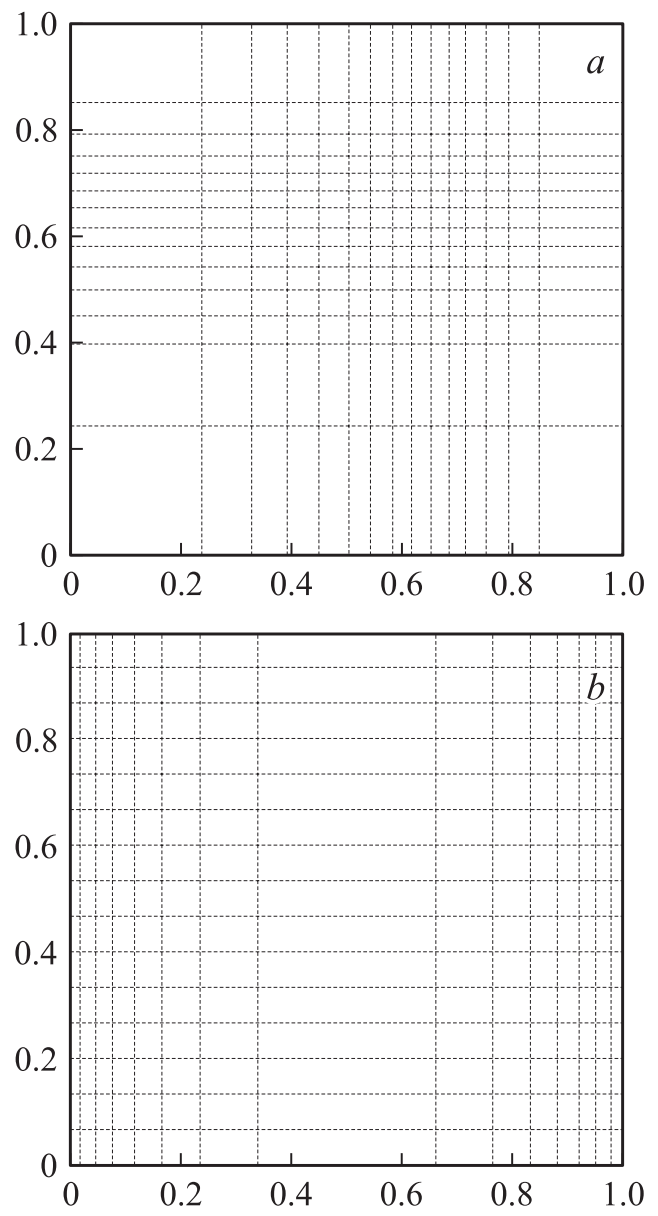

Figure 6. Modified LHS design: for function $f_{1}(a)$ and for function $f_{2}(b)$.

As a response surface model we will apply the widely used kriging interpolation method [25]. Then, we will compare obtained surfaces accouding to the following criteria - „hitting the target" (i. e. the distance between the maximun of the function we are modeling and the maximum of the evaluated surface)

$$
\operatorname{Er} r_{d}=\sqrt{\left(x_{1}^{\max }-\hat{x}_{1}^{\max }\right)^{2}+\left(x_{2}^{\max }-\hat{x}_{2}^{\max }\right)^{2}}
$$

and the total mean square error (MSE) of the surface adjustment

$$
\operatorname{Er} r_{\mathrm{MSE}}=\frac{1}{N^{2}} \sum_{i, j=1}^{N}\left(f\left(x_{1}^{i}, x_{2}^{j}\right)-\hat{f}\left(x_{1}^{i}, x_{2}^{j}\right)\right)^{2}
$$

Considering the random character of the LHS design we will repeat every experiment 100 times and take the average values of the calculated criteria as a result.

To simulate the knowledge of an expert suspecting nonlinearity in certain areas we will modify the distribution of the LH sample points as shown in Fig. 6. 

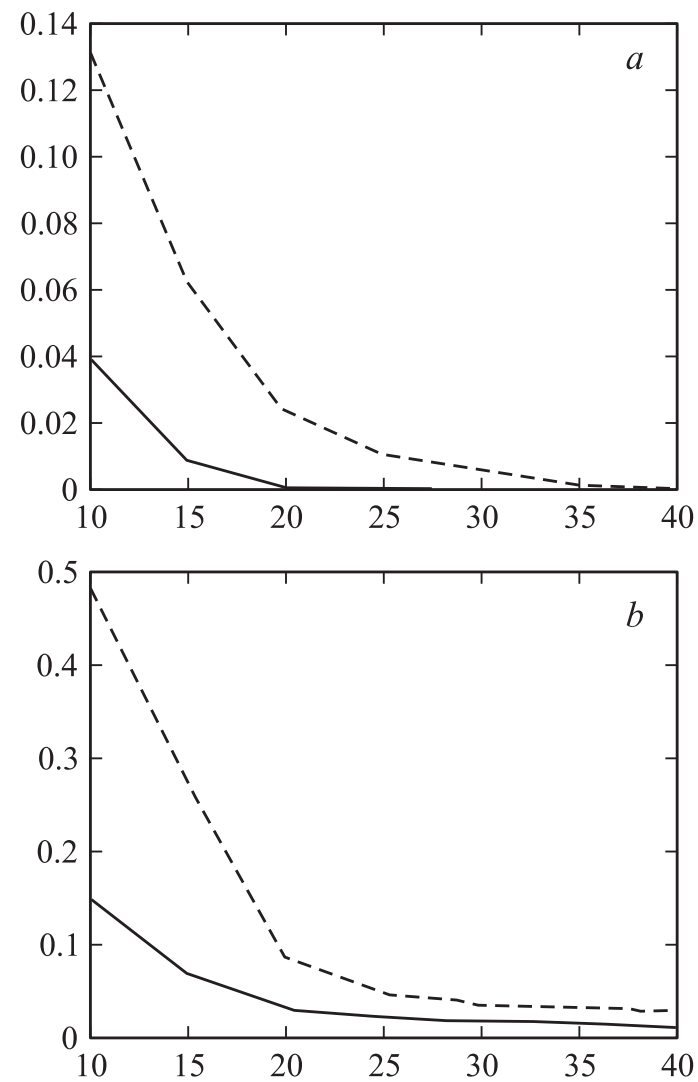

Figure 7. $\operatorname{Er}_{d}$ of standard (dashed) and modified (solid) LHS, for function $f_{1}(a)$ and for function $f_{2}(b)$.
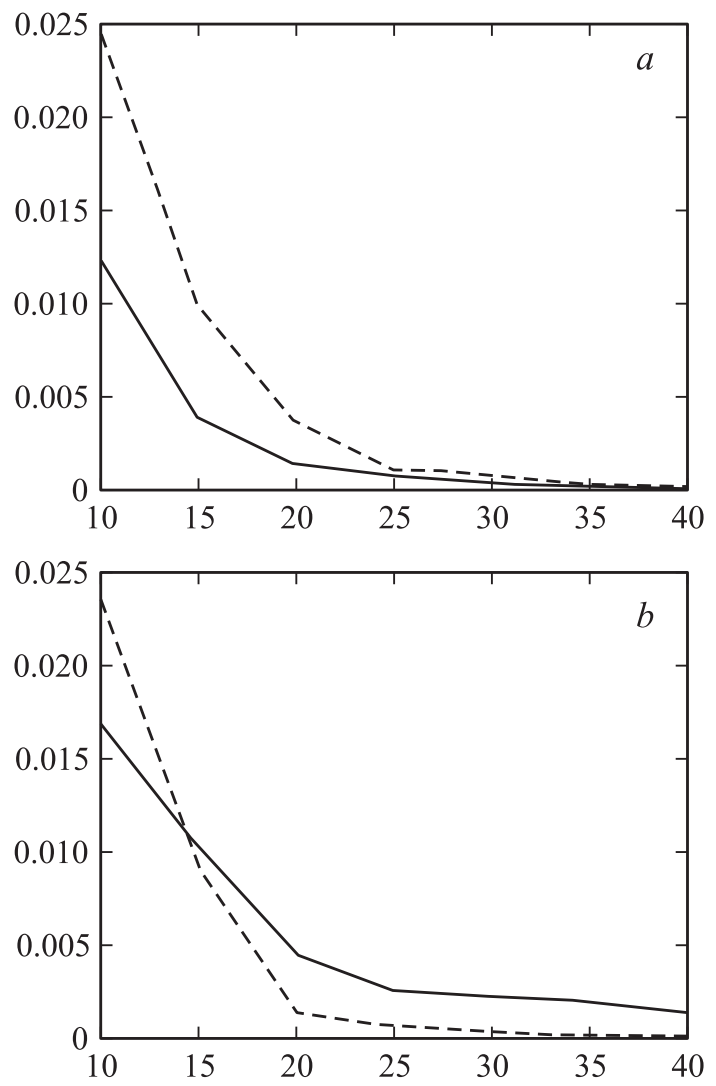

Figure 8. $\operatorname{Er} r_{\mathrm{MSE}}$ of standard (dashed) and modified (solid) LHS, for function $f_{1}(a)$ and for function $f_{2}(b)$.

\section{Criterion 1 - „hitting the target“}

This is a frequently encountered goal for an experiment. Especially when dealing with optimization it is required to properly indicate the extremum of the function. In our experiment we will focus on finding the location of the test functions' maxima. As a measure we will take the distance between the locations of real and evaluated maxima. As we can see in Fig. 7, the error of finding the maximum is significantly smaller (for both analyzed functions) if we use the modified LHS. Of course, it also decreases when we enlarge the size of the sample, still it is higher for the standard LHS.

\section{Criterion 2 - mean square error}

In this case, we will focus on the overall adjustment of the response surface. The measure will now be the mean square error. For function $f_{1}$ (single maximun) we observe better adjustment of the response surface when using the modified LHS (see Fig. 8). On the other hand, the surface obtained when using standard LHS is significantly better for function $f_{2}$. The reason is that striving for the better adjustment near the maxima of $f_{2}$ caused worse fitting in the area between.

We are indebted to Bozena Hilczer for critical reading of manuscript and attention to the work and to Sergei Vakhrushev for many helpful remarks.

\section{References}

[1] V.G. Vaks. Introduction in microscopic theory of ferroelectrics. Nauka, Moscow (1973). (in Russian).

[2] R. Blinc, B. Zeks. Soft modes in ferroelectrics and antiferroelectrics. Elsevier, N.Y. (1974).

[3] S.B. Vakhrushev, S.M. Shapiro. Phys. Rev. B 66, 214101 (2002).

[4] P.M. Gehring, S.E. Park, G. Shirane. Phys. Rev. Lett. 84, 5216 (2000).

[5] M.D. Glinchuk, V.A. Stephanovich, E.A. Eliseev, E.V. Kirichenko, L. Jastrabik. Appl. Phys. Lett. 80, 646 (2002).

[6] D. Viehland, J.F. Li, S.J. Jang, L.E. Cross. Phys. Rev. B 43, 8316 (1999).

[7] E.V. Colla, E.Yu. Koroleva, N.M. Okuneva, S.B. Vakhrushev. J. Phys.: Cond. Mat. 4, 3671 (1992).

[8] P.M. Gehring, S. Wakimoto, Z.-G. Ye, G. Shirane. Phys. Rev. Lett. 87, 277601 (2001); D. La-Orauttapong, B. Noheda, Z.G. Ye, P.M. Gehring, J. Toulouse, D.E. Cox, G. Shirane. Phys. Rev. B 65, 144101 (2002); S. Wakimoto, C. Stock, Z.-G. Ye, W. Chen, P.M. Gehring, G. Shirane. Phys. Rev. B 66, 224102 (2002).

[9] P.M. Gehring, S.E. Park, G. Shirane. Phys. Rev. B 63, 224109 (2001).

[10] J. Harada, J.D. Axe, G. Shirane. Phys. Rev. B 4, 155 (1971).

[11] J. Hlinka, S. Kamba, J. Petzelt, J. Kulda, C.A. Randall, S.J. Zhang. Phys. Rev. Lett. 91, 107602 (2003); J. Hlinka, S. Kamba, J. Petzelt, J. Kulda, C.A. Randall, S.J. Zhang. J. Phys.: Cond. Mat. 15, 4249 (2003).

[12] B.E. Vugmeister, M.D. Glinchuk. Rev. Mod. Phys. 62, 993 (1990). 
[13] M.D. Glinchuk, V.A. Stephanovich. J. Phys.: Cond. Mat. 6, 6317 (1994).

[14] M.D. Glinchuk, V.A. Stephanovich. J. Phys.: Cond. Mat. 10, 11081 (1998).

[15] M.D. Glinchuk, V.A. Stephanovich. J. Appl. Phys. 85, 1722 (1999).

[16] M.D. Glinchuk, R. Farhi. J. Phys.: Cond. Mat. 8, 6985 (1996).

[17] M.D. McKay, R.J. Bechman, W.J. Conover. Technometrics 21, 239 (1979).

[18] Ch. Kittel Quantum theory of Solids. Wiley, N.Y. (1973).

[19] P.W. Anderson, B.I. Halperin, C.M. Varma. Phil. Mag. 25, 1 (1972).

[20] R.L. Iman, W.J. Conover. Communication Statistics - Theory and Methods A 9, 1749 (1980).

[21] J. Sachs, S.B. Schiller, W.J. Welch. Technometrics 31, 41 (1989).

[22] B. Tang. J. Amer. Stat. Assoc. 88, 1392 (1993).

[23] J.S. Park. J. Statistical Planning and Interferences 90, 145 (1994).

[24] K.Q. Ye, W. Li, A. Sudianto. J. Statistical Planning and Interferences 90, 145 (2000).

[25] Noel A.C. Cressie. Statistics for Spatial Data. J. Wiley, N. Y. (1993). 